\author{
Satie Ledoux Takeda Berger \\ Universidade Federal de Santa Catarina (UFSC) \\ satietakeda@hotmail.com
}

Guilherme Luz Tortorella

Universidade Federal de Santa Catarina (UFSC)

\title{
Lean supply chain management: uma revisão sistemática da literatura das principais práticas, barreiras e fatores contextuais inerentes à sua implementação
}

\section{Resumo}

Com a intensa competição de negócios, muitas empresas têm buscado soluções para realizar suas atividades de forma mais eficiente em comparação com seus concorrentes. Se no passado a competição era entre empresas, hoje este paradigma ocorre entre a cadeia de suprimentos da empresa e a cadeia de suprimentos de seus concorrentes. Nesse contexto, a aplicação dos princípios da produção enxuta difundiu-se a diversos segmentos e organizações, estendendo-se também para a cadeia de suprimentos, onde recebeu a denominação de Lean Supply Chain Management (LSCM ou Gestão da cadeia de suprimentos enxuta). O objetivo deste artigo é realizar uma análise sistemática da literatura para identificar as principais práticas de LSCM, as barreiras para tal implementação e os fatores contextuais que a influenciam. Este artigo busca contribuir para o campo de construção da teoria do LSCM, proporcionando um maior conhecimento da literatura existente, bem como evidenciar lacunas de pesquisas para fomentar estudos futuros.

Palavras-chave: LSCM, Práticas enxutas, Barreiras, Fatores contextuais.

\begin{abstract}
With the intense competition, many companies have sought solutions to perform their activities more efficiently compared to their competitors. If in the past the competition was between companies, today this paradigm occurs between the supply chain of the company and the supply chain of its competitors. In this context, the application of lean production principles spread to several segments and organizations, including the supply chain, where it received the name of Lean Supply Chain Management (LSCM). The objective of this paper is to perform a systematic literature review to identify the main LSCM practices, the barriers to such implementation and the contextual factors that influence it. This article aims to contribute to the construction of the LSCM theory, providing a better knowledge of the existing literature, as well as evidence of research gaps to encourage future studies.
\end{abstract}

Keywords: LSCM, Lean practices, Barriers, Contextual factors.

\section{Introdução}

A cadeia de suprimentos abrange todas as atividades relacionadas com o fluxo e transformação de produtos iniciando desde a matéria-prima até o usuário final, contemplando os 
fluxos de materiais e informações que fluem tanto para baixo quanto para cima na cadeia de suprimentos (BALLOU, 2006). Segundo Ugochukwu et al. (2012) e Christopher e Towill (2001), o adequado Gerenciamento da Cadeia de Suprimentos (SCM, Supply Chain Management) é um fator chave para as empresas e influencia no desempenho das atividades de manufatura trazendo benefícios amplamente reconhecidos, como: baixo estoque, satisfação do cliente, eficiência otimizada, alta qualidade, redução de custo e melhoria no tempo de entrega e nas especificações de quantidade e qualidade e alta flexibilidade. Alves Filho et al. (2004) enfatizam a crescente quantidade de evidências de pesquisas que analisam as situações e as práticas implementadas para a gestão de cadeias de suprimentos, com abordagens diversificadas abrangendo ambos os fluxos de material e informação.

Jasti e Kodali (2015) enfatizam que para qualquer organização sobreviver é preciso produzir os produtos certos, com qualidade e quantidade, no preço e tempo certos, para o cliente certo. Dentro desse cenário, devido os benefícios proporcionados aos ambientes manufatureiros, a incorporação dos princípios e práticas enxutas na SCM vem culminando em resultados diferenciados ao longo da cadeia de suprimentos, superando aqueles já atingidos individualmente pelas organizações (ARIF-UZZAMAN; AHSAN, 2014; AFONSO; CABRITA, 2015). Nesse sentido, a frequente extensão da aplicação dos princípios e práticas enxutas às cadeias de suprimentos recebeu a denominação de Lean Supply Chain Management (LSCM, ou Gerenciamento da Cadeia de Suprimentos Enxuta) (REUL, 2015). Vitasek et al. (2005) definem LSCM como sendo um conjunto de organizações ligadas diretamente por fluxos à montante e à jusante de produtos, serviços, finanças e informação que trabalham de forma colaborativa visando a redução de custos e desperdícios, evidenciando de modo eficiente o que é necessário para atender as necessidades individuais do cliente. Para Shah e Ward (2007), o LSCM enfatiza o uso das práticas enxutas de maneira sinérgica, de modo a criar um sistema produtivo de alta qualidade, que produz conforme a demanda do cliente com pouco ou nenhum desperdício.

Muitos estudos podem ser encontrados abordando princípios e práticas enxutas na cadeia de suprimentos (LEVY, 1997; SRIDHARAN et al., 2005; TAYLOR, 2006; LI et al., 2006; BOONSTHONSATIT; JUNGTHAWAN, 2015), porém, a maioria dessas pesquisas restringe-se a aplicação de práticas em determinados segmentos de indústrias; ou abordam isoladamente algumas práticas enxutas, negligenciando a perspectiva holística da aplicação de tais práticas na SCM. Nesse contexto, Jasti e Kodali (2015) comentam a inexistência de uma teoria estável e unidirecional relativa aos conceitos do LSCM, visto que muitos estudos incidem apenas sobre aspectos individuas do LSCM, e poucos possuem foco em ambas as atividades a montante e à jusante do sistema da organização.

Para Anand e Kodali (2008) diversas modificações devem ser realizadas para adaptar os princípios e práticas enxutas para a LSCM. Enquanto a manufatura envolve predominantemente o fluxo de materiais juntamente com uma quantidade reduzida de informação dentro dos limites da organização; a cadeia de suprimentos compreende o fluxo de materiais, informação e recursos para além dos limites da organização. Assim, tanto os benefícios quanto as barreiras enfrentadas para implementação do LSCM podem divergir daqueles já conhecidos na manufatura (MANZOURI; RAHMAN, 2013). Soma-se a isso o fato de que os fatores contextuais que influenciam a implementação do LSCM (p.ex. setor e tamanho da cadeia, nível em que a empresa se encontra na cadeia, entre outros) ainda são pouco evidenciados na literatura, se comparados aos estudos em ambientes de manufatura (LI et al., 2006).

De um modo geral, verifica-se a incipiência da literatura com relação ao tema abordado e, consequentemente, três questões de pesquisa são propostas: (i) Quais as principais práticas de LSCM?; (ii) Quais as barreiras inerentes à sua implementação?; e (iii) Quais os fatores contextuais relevantes para a implementação do LSCM?. Assim, o objetivo deste artigo é realizar uma análise sistemática da literatura para identificar as principais práticas de LSCM, as barreiras para tal implementação e os fatores contextuais que a influenciam. Logo, por meio dessa revisão sistemática da literatura, espera-se identificar as principais práticas de LSCM e as barreiras e fatores contextuais inerentes à sua implementação presentes na literatura, discutir a respeito da relevância de pesquisas nesse tema e apresentar as lacunas e direcionamentos para pesquisas futuras, os quais visam complementar o corpo de conhecimento acerca do tema.

Para melhor compreensão da pesquisa, este artigo está estruturado da seguinte maneira: na seção 2.2 é apresentado o método de pesquisa proposto, em que são detalhados os procedimentos 
adotados; na seção 2.3 são apresentadas as práticas de LSCM; a seção 2.4 traz as barreiras para implementação do LSCM; a seção 2.5 consolida as variáveis contextuais que influenciam a implementação do LSCM; e finalmente, na seção 2.6 são expostas as considerações finais e direcionamentos para pesquisa futuras.

\section{Procedimentos metodológicos}

Para a realização deste trabalho adotou-se a revisão sistemática de literatura. Esta utiliza a aplicação de métodos explícitos e sistematizados de busca, síntese da informação selecionada, e integra as informações de um conjunto de estudos realizados separadamente sobre determinado assunto (SAMPAIO; MANCINI, 2007; PARÉ et al., 2015). As etapas do método adotado, estão descritas na Tabela 1 e serão detalhadas a seguir.

Tabela 1 - Descrição geral sobre o processo de revisão sistemática da literatura

\begin{tabular}{cl} 
Etapas & \multicolumn{1}{c}{ Descrição } \\
\hline 1 & Definir a questão de pesquisa, abordando o tema de interesse \\
\hline 2 & $\begin{array}{l}\text { Identificar as bases de dados a serem consultadas; definir palavras-chave e estratégias de } \\
\text { busca }\end{array}$ \\
\hline 3 & Estabelecer critérios para a seleção dos artigos a partir da busca \\
\hline 4 & Conduzir busca nas bases de dados escolhidas e com base na (s) estratégia (s) definidas (s) \\
\hline 5 & Definir o portfólio inicial de artigos \\
\hline 6 & Aplicar os critérios na seleção dos artigos e justificar possíveis exclusões \\
\hline 7 & Analisar criticamente e avaliar todos os estudos na revisão \\
\hline 8 & $\begin{array}{l}\text { Preparar um resumo, sintetizando as informações disponibilizadas pelos artigos } \\
\text { selecionados }\end{array}$ \\
\hline 9 & Apresentar uma conclusão, informando a evidência sobre os efeitos da intervenção \\
\hline
\end{tabular}
Fonte: Adaptado de Sampaio e Mancini (2007)

Na primeira etapa é definida a questão de pesquisa, a qual o trabalho buscará responder e servirá de norte para o início da revisão sistemática da literatura. Este estudo é composto por três questões já apresentadas na sua seção introdutória. Em seguida, na segunda etapa, foram definidas as bases de dados que foram utilizadas para a busca dos artigos. As bases foram escolhidas conforme a disponibilidade no Portal Periódicos CAPES (Coordenação de Aperfeiçoamento de Pessoal de Nível Superior), utilizando os filtros de bases que fossem da área de conhecimento das Engenharias e subárea Engenharia de Produção. As estratégias definidas para a escolha das bases de dados foram delimitadas segundo Lancaster (2004), o qual alega que uma base de dados bibliográficos deve ser avaliada pela sua utilidade ao responder as seguintes necessidades de informação: a) cobertura, o quão completo é o conteúdo da base de dados em relação a um assunto; b) recuperabilidade, quantos documentos sobre o assunto podem ser encontrados na base utilizando-se uma estratégia de busca não muito complexa; c) previsibilidade, o pesquisador consegue verificar com eficiência a relevância dos documentos a partir das informações contidas na base de dados; e d) atualidade, a base de dados possui uma boa frequência na inclusão de novas publicações. Desta forma, definiu-se três bases para a consulta: Emerald, Scopus e Web of Science. Ainda nesta etapa, para a busca dos artigos nas bases estabelecidas foram definidas as combinações de palavras-chave, conforme Quadro 1.

$\mathrm{Na}$ terceira etapa, foram estabelecidos alguns critérios para a seleção dos artigos encontrados, sendo eles: (a) somente pesquisas do tipo artigo científico; (b) títulos dos artigos alinhados ao tema de pesquisa; (c) resumos alinhados ao tema de pesquisa; (d) texto integral dos artigos alinhados com o tema da pesquisa; e (e) sem delimitação temporal das publicações. Na etapa quatro, com base nas palavra-chave selecionadas, foi realizada uma busca nas bases de dados escolhidas de acordo com as estratégias definidas anteriormente. Na etapa cinco, foi definido o portfólio inicial de análise, o qual totalizou 1.384 referências. Além disso, efetuou-se a exportação 
dessas referências para a ferramenta de gerenciamento EndNote $X 7 \AA$ de modo a auxiliar na sua organização. Posteriormente, na etapa seis, os critérios definidos na etapa três foram aplicados na seleção de artigos, de modo a excluir aqueles que não estavam alinhados com os objetivos. Nesta etapa obteve-se a exclusão de 867 artigos duplicados ou de natureza não científica, além de 457 artigos sem relação com o tema, através da análise dos títulos, resumos ou texto integral. Ao total, foram removidos 1.323 trabalhos, restando apenas 60. Em sequência, na etapa sete, foi realizada uma leitura crítica e avaliação do portfólio final de artigos definido; para em seguida, na etapa oito, desenvolver um resumo crítico composto pelas principais informações relevantes disponibilizadas pelos artigos incluídos na revisão e, então, apresentar as considerações e contribuições desse estudo que foi realizado (etapa 9).

Quadro 1 - Levantamento bibliográfico da pesquisa e seleção dos artigos

\begin{tabular}{|c|c|c|c|}
\hline \multirow[b]{2}{*}{ Palavras-chave } & \multicolumn{3}{|c|}{ Quantitativo das bases de dados } \\
\hline & Emerald & Scopus & $\begin{array}{l}\text { Web of } \\
\text { Science }\end{array}$ \\
\hline "lean supply" E "practices" OU “implement" & 234 & 58 & 25 \\
\hline "lean supply" E "failures" OU “challenges" OU "barriers" & 175 & 40 & 8 \\
\hline "lean supply" E "contextual factors" & 189 & 8 & 18 \\
\hline "lean supply" & 282 & 236 & 111 \\
\hline Total de cada base & 880 & 343 & 162 \\
\hline Total geral (Portfólio Inicial) & \multicolumn{3}{|c|}{1.384} \\
\hline Artigos duplicados ou não científicos & \multicolumn{3}{|c|}{867} \\
\hline $\begin{array}{c}\text { Artigos sem relação com os objetivos do trabalho (título e } \\
\text { resumo) }\end{array}$ & \multicolumn{3}{|c|}{401} \\
\hline $\begin{array}{l}\text { Artigos sem relação com os objetivos do trabalho (texto } \\
\text { integral) }\end{array}$ & \multicolumn{3}{|c|}{56} \\
\hline Total de artigos selecionados (Portfólio Final) & \multicolumn{3}{|c|}{60} \\
\hline
\end{tabular}

\section{Resultados}

\subsection{Práticas de LSCM}

As práticas enxutas podem ser aplicadas em toda a extensão da cadeia de suprimentos, desde a colocação do pedido com os fornecedores até a distribuição e entrega do produto ao cliente final. Estudos anteriores (WEE, WU, 2009; PEREZ et al., 2010; VLACHOS, 2015; BOONSTHONSATIT; JUNGTHAWAN, 2015) associam a implementação das práticas de LSCM com melhorias no desempenho operacional da cadeia, independentemente do contexto em estudo. Erridge e Murray (1998), por exemplo, evidenciam que, através da aplicação das práticas de LSCM, benefícios similares aos da indústria de transformação podem ser obtidos na cadeia de suprimentos da Câmara Municipal de Belfast, principal cidade da Irlanda do Norte, tais como redução de estoque, aumento de qualidade nos serviços, redução de custos e melhor relacionamento com fornecedores e clientes. No entanto, estudos que tratem da aplicação das práticas de LSCM ainda são menos frequentemente evidenciados na literatura, visto que a expansão do pensamento enxuto para o contexto da cadeia de suprimentos mostra-se mais complexo (MARTÍNEZ-JURADO; MOYANO-FUENTES, 2014).

Dentre os artigos revisados, apenas dois estudos propuseram estruturas conceituais amplas referentes à implementação das práticas de LSCM. Soni e Kodali (2008), complementado posteriormente por Jasti e Kodali (2015), sugerem oito pilares para a implementação de LSCM, sendo estes constituídos por oitenta e duas práticas; são eles: ( $i$ ) gestão da tecnologia da informação; (ii) gestão de fornecedores; (iii) eliminação de desperdícios; (iv) produção JIT; ( $v$ ) gestão de 
relacionamento com o cliente; (vi) gestão de logística; (vii) comprometimento da alta gestão; (viii) melhoria contínua. No entanto, a estrutura conceitual proposta não foi empiricamente validada, caracterizando uma lacuna de pesquisa.

A Tabela 2 consolida as práticas (P) de LSCM mais citadas na literatura de acordo com 55 estudos elencados a partir da revisão sistemática da literatura, havendo uma variação significativa na frequência de citação de cada $\mathrm{P}$. As práticas $\mathrm{P}_{1}$ (Produção puxada ou Kanban) e $\mathrm{P}_{2}$ (Relacionamento estreito e de longo prazo entre fornecedores, clientes e demais partes envolvidas) aparecem como as mais frequentemente citadas nos estudos de LSCM, sendo 38 e 32 vezes respectivamente. Essas altas frequências de citações podem ser explicadas devido ao fato dessas $\mathrm{P}$ serem incluídas nos estudos precursores de LSCM, dado que impactam tanto o processo fabril quanto o desempenho da cadeia de suprimentos (LAMMING, 1996). Na verdade, estas P foram consistentemente associadas aos estudos de LSCM ao longo do tempo, acarretando em um alto número de evidências que reportem seu uso. Especificamente para a $\mathrm{P}_{1}$, McIvor (2001) comenta seu impacto sobre a obtenção de menores níveis de estoque e maior visibilidade de problemas de qualidade. Além disso, tal $\mathrm{P}$ é comumente associada à entregas just-in-time (JIT) (WIENGARTEN et al., 2013; DÜES et al. 2013), nas quais o material certo é entregue no momento, lugar e quantidade esperados (QRUNFLEH; TARAFDAR, 2013). Consequentemente, a adoção de $\mathrm{P}_{1}$ implica no estreitamento dos fluxos de informação e material entre fornecedores e clientes, reforçando a colaboração entre ambos $\left(\mathrm{P}_{2}\right)$ (MARTÍNEZ-JURADO; MOYANO-FUENTES, 2014). Nesse sentido, é razoável esperar-se que ambos $\mathrm{P}_{1}$ e $\mathrm{P}_{2}$ apresentem alta recorrência de citações ao longo do tempo, uma vez que estão intimamente relacionadas (BHAMU; SINGH SANGWAN, 2014).

Por outro lado, a $\mathrm{P}_{18}$ (Formação de centros de distribuição) mostrou-se como a menos referida na literatura, apresentando somente 3 citações dentre os 55 estudos. A implementação de centros de distribuição geralmente é motivada devido potenciais impactos sobre os custos de transporte e processamento de pedidos (BAKER, 2004). Apesar dos primeiros estudos sobre a prática de formação de centros de distribuição datarem da década de 70 (LA LONDE et al., 1971), sua associação e posterior inserção na abordagem de LSCM é relativamente mais recente. De fato, Taylor (2006) tangencia a incorporação dessa prática no contexto de implementação de LSCM. Contudo, somente em Sharma et al. (2015) e Jasti e Kodali (2015) que esta foi explicitamente incluída no conjunto de práticas de LSCM. Assim, a partir da maior compreensão e expansão do pensamento enxuto para as cadeias de suprimentos, o qual proporcionou um enfoque muito mais abrangente quanto a implementação do LSCM, a $\mathrm{P}_{18}$ ganhou considerável atenção e começou a ser tratada como uma prática de LSCM. 
Tabela 2 - Práticas de LSCM e sua frequência de citação na literatura (Parte I)

\begin{tabular}{|c|c|c|c|c|c|c|c|c|c|c|c|c|c|c|c|c|c|c|c|c|c|}
\hline & Autores & 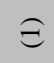 & d & $\widehat{(2)}$ & 丹 & $\approx$ & e & $E$ & $\widehat{\infty}$ & $\widehat{\varrho}$ & $\stackrel{\varrho}{\varrho}$ & $\Xi$ & $\widehat{\Xi}$ & $\stackrel{\overbrace{}}{=}$ & $\stackrel{\Im}{\Xi}$ & $\cong$ & $\stackrel{6}{=}$ & $\cong$ & $\stackrel{\infty}{=}$ & $\stackrel{\varrho}{\varrho}$ & ¿े \\
\hline $\mathrm{P}_{1}$ & Produção puxada ou kanban & $\mathrm{x}$ & $\mathrm{x}$ & & $\mathrm{x}$ & & & $\mathrm{x}$ & & $\mathrm{x}$ & & & $\mathrm{x}$ & $\mathrm{x}$ & $\mathrm{x}$ & $\mathrm{x}$ & & $\mathrm{x}$ & $\mathrm{x}$ & $\mathrm{x}$ & $\mathrm{x}$ \\
\hline $\mathrm{P}_{2}$ & Relacionamento estreito e de longo prazo entre fornecedores, clientes e demais partes envolvidas & $\mathrm{x}$ & $\mathrm{x}$ & & $\mathrm{x}$ & $\mathrm{x}$ & $\mathrm{x}$ & & & $\mathrm{x}$ & & $\mathrm{x}$ & $\mathrm{x}$ & & $\mathrm{x}$ & $\mathrm{x}$ & $\mathrm{x}$ & & & $\mathrm{x}$ & $\mathrm{x}$ \\
\hline $\mathrm{P}_{3}$ & $\begin{array}{l}\text { Utilização de tecnologia da informação para compartilhamento e integração do fluxo de } \\
\text { informação ao longo da cadeia de suprimentos (p.ex.: EDI, RFID, ERP, etc.) }\end{array}$ & & $\mathrm{x}$ & & $\mathrm{x}$ & & $\mathrm{x}$ & $\mathrm{x}$ & $\mathrm{x}$ & $\mathrm{x}$ & & & $\mathrm{x}$ & $\mathrm{x}$ & $\mathrm{x}$ & & $\mathrm{x}$ & & $\mathrm{x}$ & & $\mathrm{x}$ \\
\hline $\mathrm{P}_{4}$ & Reabastecimento contínuo & $\mathrm{x}$ & & & & & & $\mathrm{x}$ & & $\mathrm{x}$ & & & & $\mathrm{x}$ & & & & $\mathrm{x}$ & $\mathrm{x}$ & $\mathrm{x}$ & $\mathrm{x}$ \\
\hline $\mathrm{P}_{5}$ & Melhoria contínua & & $\mathrm{x}$ & & & $\mathrm{x}$ & & & & & & & $\mathrm{x}$ & & $\mathrm{x}$ & $\mathrm{x}$ & & $\mathrm{x}$ & & & $\mathrm{x}$ \\
\hline $\mathrm{P}_{6}$ & Mapeamento do Fluxo de Valor & & & & & & & & & $\mathrm{x}$ & $\mathrm{x}$ & & & $\mathrm{x}$ & $\mathrm{x}$ & & & $\mathrm{x}$ & & & \\
\hline $\mathrm{P}_{7}$ & $\begin{array}{l}\text { Keiretsu (relacionamento baseado na confiança, cooperação e apoio educacional com os } \\
\text { fornecedores, desempenhando um papel estratégico importante na organização) }\end{array}$ & $\mathrm{x}$ & $\mathrm{x}$ & & & & & & $\mathrm{x}$ & $\mathrm{x}$ & & & $\mathrm{x}$ & & $\mathrm{x}$ & & $\mathrm{x}$ & & $\mathrm{x}$ & $\mathrm{x}$ & \\
\hline $\mathrm{P}_{8}$ & Estoque gerenciado pelo fornecedor (consignado) & $\mathrm{x}$ & & & & $\mathrm{x}$ & & & & $\mathrm{x}$ & & & $\mathrm{x}$ & & $\mathrm{x}$ & & $\mathrm{x}$ & & & $\mathrm{x}$ & \\
\hline $\mathrm{P}_{9}$ & Logísticas de distribuição & $\mathrm{x}$ & & & $\mathrm{x}$ & & & & & & & $\mathrm{x}$ & & $\mathrm{x}$ & $\mathrm{x}$ & & $\mathrm{x}$ & & & & \\
\hline $\mathrm{P}_{10}$ & Padronização de processos e produtos para garantia da qualidade & & & & & & & & & & & $\mathrm{x}$ & $\mathrm{x}$ & & $\mathrm{x}$ & & & $\mathrm{x}$ & & & $\mathrm{x}$ \\
\hline $\mathrm{P}_{11}$ & $\begin{array}{l}\text { Participação frequente desde o início do projeto e processo de desenvolvimento de novos } \\
\text { produtos }\end{array}$ & & $\mathrm{x}$ & $\mathrm{x}$ & $\mathrm{x}$ & & $\mathrm{x}$ & & & & & & $\mathrm{x}$ & & & & & & & & $\mathrm{x}$ \\
\hline $\mathrm{P}_{12}$ & Hoshin Kanri (desdobramento das estratégias e comprometimento e suporte da alta gestão) & $\mathrm{x}$ & & & & $\mathrm{x}$ & & & & $\mathrm{x}$ & $\mathrm{x}$ & $\mathrm{x}$ & $\mathrm{x}$ & & & & & & & & $\mathrm{x}$ \\
\hline $\mathrm{P}_{13}$ & Desenvolvimento de KPIs da cadeia de suprimentos & & & & & & & & $\mathrm{x}$ & $\mathrm{x}$ & & $\mathrm{x}$ & & & & $\mathrm{x}$ & & & & & $\mathrm{x}$ \\
\hline $\mathrm{P}_{14}$ & Nivelamento da produção ou Heijunka & $\mathrm{x}$ & & & & & & & & & & & & $\mathrm{x}$ & & & & $\mathrm{x}$ & & & \\
\hline $\mathrm{P}_{15}$ & $\begin{array}{l}\text { Kyoryoku Kai (associação de fornecedores que trabalham juntos para desenvolver métodos mais } \\
\text { eficientes de trabalho reduzindo os desperdícios) }\end{array}$ & $\mathrm{x}$ & & & & & & & & $\mathrm{x}$ & & & & & $\mathrm{x}$ & & & & & & $\mathrm{x}$ \\
\hline $\mathrm{P}_{16}$ & $\begin{array}{l}\text { Solução de problemas (feedback frequente trabalhando em conjunto para soluções } \\
\text { compartilhadas) }\end{array}$ & & & & $\mathrm{x}$ & & & & & & & & & & & & & & & $\mathrm{x}$ & \\
\hline $\mathrm{P}_{17}$ & Gerenciamento da cadeia de valor & & & & & & & & & $\mathrm{x}$ & & & & $\mathrm{x}$ & $\mathrm{x}$ & & & & & & $\mathrm{x}$ \\
\hline $\mathrm{P}_{18}$ & Formação de centros de distribuição & & & & & & & & & $\mathrm{x}$ & & & & & & & & & & & \\
\hline
\end{tabular}


Tabela 2 - Práticas de LSCM e sua frequência de citação na literatura (Parte II)

\begin{tabular}{|c|c|c|c|c|c|c|c|c|c|c|c|c|c|c|c|c|c|c|c|c|c|}
\hline & Autores & $\widehat{\bar{d}}$ & త్ & $\widetilde{\beth}$ & $\stackrel{\text { d }}{d}$ & 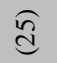 & 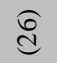 & $\widehat{\widehat{d}}$ & 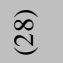 & ఏ્d & હ્હે & $\widehat{\bar{c}}$ & శ్ర & હ્ర & 宓 & ઈీ & $\begin{array}{l}\text { d) } \\
\text { (2) }\end{array}$ & $\underset{\tilde{\sigma}}{\widehat{\sigma}}$ & $\stackrel{\infty}{\stackrel{్}{C}}$ & ఏ્ల & 主 \\
\hline $\mathrm{P}_{1}$ & Produção puxada ou kanban & $\mathrm{x}$ & $\mathrm{x}$ & & $\mathrm{x}$ & $\mathrm{x}$ & & $\mathrm{x}$ & $\mathrm{x}$ & $\mathrm{x}$ & $\mathrm{x}$ & & $\mathrm{x}$ & & $\mathrm{x}$ & $\mathrm{x}$ & & $\mathrm{x}$ & $\mathrm{x}$ & $\mathrm{x}$ & $\mathrm{x}$ \\
\hline $\mathrm{P}_{2}$ & Relacionamento estreito e de longo prazo entre fornecedores, clientes e demais partes envolvidas & & $\mathrm{x}$ & $\mathrm{x}$ & & & $\mathrm{x}$ & & $\mathrm{x}$ & $\mathrm{x}$ & & & $\mathrm{x}$ & $\mathrm{x}$ & $\mathrm{x}$ & $\mathrm{x}$ & $\mathrm{x}$ & $\mathrm{x}$ & $\mathrm{x}$ & & $\mathrm{x}$ \\
\hline $\mathrm{P}_{3}$ & $\begin{array}{l}\text { Utilização de tecnologia da informação para compartilhamento e integração do fluxo de } \\
\text { informação ao longo da cadeia de suprimentos (p.ex.: EDI, RFID, ERP, etc.) }\end{array}$ & $\mathrm{x}$ & & $\mathrm{x}$ & & & $\mathrm{x}$ & & $\mathrm{x}$ & $\mathrm{x}$ & & & & & $\mathrm{x}$ & & & & $\mathrm{x}$ & & $\mathrm{x}$ \\
\hline $\mathrm{P}_{4}$ & Reabastecimento contínuo & $\mathrm{x}$ & $\mathrm{x}$ & & & $\mathrm{x}$ & & & $\mathrm{x}$ & $\mathrm{x}$ & & $\mathrm{x}$ & & & $\mathrm{x}$ & $\mathrm{x}$ & & $\mathrm{x}$ & $\mathrm{x}$ & & \\
\hline $\mathrm{P}_{5}$ & Melhoria contínua & & $\mathrm{x}$ & & & & & & & $\mathrm{x}$ & $\mathrm{x}$ & & $\mathrm{x}$ & $\mathrm{x}$ & & & & $\mathrm{x}$ & $\mathrm{x}$ & & $\mathrm{x}$ \\
\hline $\mathrm{P}_{6}$ & Mapeamento do Fluxo de Valor & & $\mathrm{x}$ & & $\mathrm{x}$ & & & $\mathrm{x}$ & & $\mathrm{x}$ & $\mathrm{x}$ & & & & & $\mathrm{x}$ & & $\mathrm{x}$ & $\mathrm{x}$ & $\mathrm{x}$ & \\
\hline $\mathrm{P}_{7}$ & $\begin{array}{l}\text { Keiretsu (relacionamento baseado na confiança, cooperação e apoio educacional com os } \\
\text { fornecedores, desempenhando um papel estratégico importante na organização) }\end{array}$ & & & $\mathrm{x}$ & & $\mathrm{x}$ & $\mathrm{x}$ & & $\mathrm{x}$ & & & & $\mathrm{x}$ & & & $\mathrm{x}$ & $\mathrm{x}$ & & & & \\
\hline $\mathrm{P}_{8}$ & Estoque gerenciado pelo fornecedor (consignado) & $\mathrm{x}$ & & & & $\mathrm{x}$ & $\mathrm{x}$ & & & & & & $\mathrm{x}$ & $\mathrm{x}$ & & $\mathrm{x}$ & & & & $\mathrm{x}$ & $\mathrm{x}$ \\
\hline $\mathrm{P}_{9}$ & Logísticas de distribuição & & & $\mathrm{x}$ & & & $\mathrm{x}$ & $\mathrm{x}$ & & $\mathrm{x}$ & & & $\mathrm{x}$ & & & & & & & & \\
\hline $\mathrm{P}_{10}$ & Padronização de processos e produtos para garantia da qualidade & & & & $\mathrm{x}$ & & & & & & & & & & & & & $\mathrm{x}$ & $\mathrm{x}$ & $\mathrm{x}$ & $\mathrm{x}$ \\
\hline $\mathrm{P}_{11}$ & $\begin{array}{l}\text { Participação freqüente desde o início do projeto e processo de desenvolvimento de novos } \\
\text { produtos }\end{array}$ & & & $\mathrm{x}$ & & & & & & & & & & $\mathrm{x}$ & & & $\mathrm{x}$ & & & & \\
\hline $\mathrm{P}_{12}$ & Hoshin Kanri (desdobramento das estratégias e comprometimento e suporte da alta gestão) & & & & & & $\mathrm{x}$ & & & & & & & & & & $\mathrm{x}$ & $\mathrm{x}$ & & & \\
\hline $\mathrm{P}_{13}$ & Desenvolvimento de KPIs da cadeia de suprimentos & & & & & $\mathrm{x}$ & & & & & $\mathrm{x}$ & & & & & $\mathrm{x}$ & & & & & \\
\hline $\mathrm{P}_{14}$ & Nivelamento da produção ou Heijunka & & & & $\mathrm{x}$ & & & & & & & & & & & & & $\mathrm{x}$ & $\mathrm{x}$ & & \\
\hline $\mathrm{P}_{15}$ & $\begin{array}{l}\text { Kyoryoku Kai (associação de fornecedores que trabalham juntos para desenvolver métodos mais } \\
\text { eficientes de trabalho reduzindo os desperdícios) }\end{array}$ & & & & & & & & $\mathrm{x}$ & & & & & & & & & & & & \\
\hline $\mathrm{P}_{16}$ & $\begin{array}{l}\text { Solução de problemas (feedback frequente trabalhando em conjunto para soluções } \\
\text { compartilhadas) }\end{array}$ & & & & & & & & & & & & & & & & $\mathrm{x}$ & $\mathrm{x}$ & & & \\
\hline $\mathrm{P}_{17}$ & Gerenciamento da cadeia de valor & & & & & & & & & & & & & & & & & & & & \\
\hline $\mathrm{P}_{18}$ & Formação de centros de distribuição & & & & 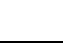 & & & & & & & & & & & & & & & & \\
\hline
\end{tabular}


Tabela 2 - Práticas de LSCM e sua frequência de citação na literatura (Parte III)

\begin{tabular}{|c|c|c|c|c|c|c|c|c|c|c|c|c|c|c|c|c|c|}
\hline & Autores & 卞 & $\stackrel{\overparen{I}}{\mathcal{I}}$ & $\widehat{\mathcal{f}}$ & 籿 & $\stackrel{\overbrace{}}{f}$ & 吕 & 导 & $\stackrel{\text { P }}{+\infty}$ & $\stackrel{\partial}{\dot{d}}$ & 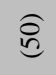 & $\sqrt{6}$ & శ్ & $\widehat{\approx n}$ & 点 & $\sqrt{n}$ & $\begin{array}{l}\text { Frequência } \\
\text { de citação }\end{array}$ \\
\hline $\mathrm{P}_{1}$ & Produção puxada ou kanban & $\mathrm{x}$ & & $\mathrm{x}$ & $\mathrm{x}$ & & $\mathrm{x}$ & $\mathrm{x}$ & $\mathrm{x}$ & & $\mathrm{x}$ & & $\mathrm{x}$ & $\mathrm{x}$ & & $\mathrm{x}$ & 38 \\
\hline $\mathrm{P}_{2}$ & Relacionamento estreito e de longo prazo entre fornecedores, clientes e demais partes envolvidas & & & $\mathrm{x}$ & $\mathrm{x}$ & & $\mathrm{x}$ & & & $\mathrm{x}$ & & & & & $\mathrm{x}$ & $\mathrm{x}$ & 32 \\
\hline $\mathrm{P}_{3}$ & $\begin{array}{l}\text { Utilização de tecnologia da informação para compartilhamento e integração do fluxo de informação ao } \\
\text { longo da cadeia de suprimentos (p.ex.: EDI, RFID, ERP, etc.) }\end{array}$ & & & $\mathrm{x}$ & $\mathrm{x}$ & $\mathrm{x}$ & & & $\mathrm{x}$ & & & & & & $\mathrm{x}$ & $\mathrm{x}$ & 26 \\
\hline $\mathrm{P}_{4}$ & Reabastecimento contínuo & $\mathrm{x}$ & & & $\mathrm{x}$ & $\mathrm{x}$ & $\mathrm{x}$ & & & & $\mathrm{x}$ & & $\mathrm{x}$ & $\mathrm{x}$ & & & 25 \\
\hline $\mathrm{P}_{5}$ & Melhoria contínua & $\mathrm{x}$ & & & $\mathrm{x}$ & $\mathrm{x}$ & $\mathrm{x}$ & $\mathrm{x}$ & $\mathrm{x}$ & & $\mathrm{x}$ & $\mathrm{x}$ & & & $\mathrm{x}$ & & 24 \\
\hline $\mathrm{P}_{6}$ & Mapeamento do Fluxo de Valor & $\mathrm{x}$ & $\mathrm{x}$ & & $\mathrm{x}$ & $\mathrm{x}$ & $\mathrm{x}$ & $\mathrm{x}$ & $\mathrm{x}$ & & $\mathrm{x}$ & & & & $\mathrm{x}$ & & 23 \\
\hline $\mathrm{P}_{7}$ & $\begin{array}{l}\text { Keiretsu (relacionamento baseado na confiança, cooperação e apoio educacional com os fornecedores, } \\
\text { desempenhando um papel estratégico importante na organização) }\end{array}$ & & & $\mathrm{x}$ & $\mathrm{x}$ & & $\mathrm{x}$ & & & $\mathrm{x}$ & & $\mathrm{x}$ & & & & & 21 \\
\hline $\mathrm{P}_{8}$ & Estoque gerenciado pelo fornecedor (consignado) & & & $\mathrm{x}$ & $\mathrm{x}$ & & $\mathrm{x}$ & & $\mathrm{x}$ & & & & & & $\mathrm{x}$ & & 20 \\
\hline $\mathrm{P}_{9}$ & Logísticas de distribuição & & & $\mathrm{x}$ & $\mathrm{x}$ & $\mathrm{x}$ & $\mathrm{x}$ & & $\mathrm{x}$ & & & $\mathrm{x}$ & & & $\mathrm{x}$ & & 18 \\
\hline $\mathrm{P}_{10}$ & Padronização de processos e produtos para garantia da qualidade & $\mathrm{x}$ & & $\mathrm{x}$ & $\mathrm{x}$ & $\mathrm{x}$ & & & & & $\mathrm{x}$ & $\mathrm{x}$ & $\mathrm{x}$ & & & & 17 \\
\hline $\mathrm{P}_{11}$ & Participação freqüente desde o início do projeto e processo de desenvolvimento de novos produtos & & & & $\mathrm{x}$ & & $\mathrm{x}$ & & & $\mathrm{x}$ & & $\mathrm{x}$ & & & & & 13 \\
\hline $\mathrm{P}_{12}$ & Hoshin Kanri (desdobramento das estratégias e comprometimento e suporte da alta gestão) & & & & $\mathrm{x}$ & & & & & & & $\mathrm{x}$ & & & & & 12 \\
\hline $\mathrm{P}_{13}$ & Desenvolvimento de KPIs da cadeia de suprimentos & & & & $\mathrm{x}$ & $\mathrm{x}$ & $\mathrm{x}$ & & & & & $\mathrm{x}$ & & & & & 12 \\
\hline $\mathrm{P}_{14}$ & Nivelamento da produção ou Heijunka & & & & $\mathrm{x}$ & $\mathrm{x}$ & $\mathrm{x}$ & & & & & & & & & $\mathrm{x}$ & 10 \\
\hline $\mathrm{P}_{15}$ & $\begin{array}{l}\text { Kyoryoku Kai (associação de fornecedores que trabalham juntos para desenvolver métodos mais } \\
\text { eficientes de trabalho reduzindo os desperdícios) }\end{array}$ & & & & $\mathrm{x}$ & & & & $\mathrm{x}$ & & & & & $\mathrm{x}$ & & & 8 \\
\hline $\mathrm{P}_{16}$ & Solução de problemas (feedback frequente trabalhando em conjunto para soluções compartilhadas) & & & & $\mathrm{x}$ & & $\mathrm{x}$ & & & & & & $\mathrm{x}$ & & & & 7 \\
\hline $\mathrm{P}_{17}$ & Gerenciamento da cadeia de valor & & & & $\mathrm{x}$ & & $\mathrm{x}$ & $\mathrm{x}$ & & & & & & & & & 7 \\
\hline $\mathrm{P}_{18}$ & Formação de centros de distribuição & & & & $\mathrm{x}$ & & $\mathrm{x}$ & & & & & & & & & & 3 \\
\hline
\end{tabular}




\subsection{Barreiras para implementação do LSCM}

Uma barreira é um obstáculo que impede ou restringe o progresso tornando difícil alcançar algo (KUMAR et al., 2016). Para se ter sucesso em qualquer mudança em uma organização, as forças de resistência ou barreiras precisam ser identificadas e compreendidas (JADHAV et al., 2014). Acredita-se que a implementação de uma estratégia enxuta, como qualquer outra iniciativa de melhoria, acarreta enormes dificuldades (RAHMAN et al., 2010). O conceito de LSCM tem sido aplicado a diversos segmentos nas últimas décadas. Porém, a razão pela qual permanece uma questão aberta é porque todo o sistema de cadeia de suprimentos é muito complexo e apresenta resultados em longo prazo, tornando um desafio realizar melhorias em toda a extensão da cadeia (ADEBANJO et al., 2016). Os estudos sobre as práticas de LSCM em diferentes segmentos permitem distinguir as particularidades das práticas aplicadas e também as barreiras enfrentadas para sua adoção (YEW WONG et al., 2005).

A partir dos 60 artigos revisados, apenas 34 abordaram algum tipo de barreira inerente à implementação do LSCM. Destes, apenas 4 artigos apresentaram explicitamente como tema principal as barreiras e desafios do LSCM (p.ex.: MCIVOR, 2001; JADHAV et al. 2014; DORA et al., 2016; MANZOURI et al., 2013a). Os demais artigos analisados, abordaram algumas barreiras de forma tangencial decorrente da aplicação das práticas de LSCM (p. ex. ARKADER, 2001; ANAND; KODALI, 2008; PEREZ et al., 2010; ADEBANJO et al., 2016). Vlachos (2015) descreve a implementação das práticas de LSCM em uma empresa de chá do Reino Unido, salientando as dificuldades encontradas. Dentre elas, o autor relata a falta de envolvimento da alta gerência no projeto, resultando em uma implementação limitada e fracassada. Jadhav et al. (2014) comentam que a única maneira de criar uma verdadeira transformação enxuta é com uma forte liderança no topo de uma organização, incluindo o diretor executivo. Para isto, seu real envolvimento é fundamental de modo que a alta gerência forneça apoio para sustentação das melhorias (YEW WONG et al., 2005). Nesse sentido, a falta de comprometimento pode levar a uma série de questões, tais como o acesso limitado aos recursos, processos de tomada de decisão prolongados e falhas de comunicação (PEREZ et al., 2010).

Na Tabela 3 foram sintetizadas as barreiras (B) inerentes à implementação do LSCM de acordo com o portfólio de artigos selecionados. Percebe-se que existe um grau variável de frequência de citação relacionado a cada uma das barreiras nos estudos revisados. A barreira $\mathrm{B}_{1}$ (Dificuldades para mudanças culturais) apareceu como uma das mais frequentemente enfrentadas durante à implementação do LSCM.

As principais dificuldades enfrentadas na implementação dos princípios e práticas enxutas estão relacionadas a questões humanas, culturais e organizacionais (JADHAV et al., 2014). A mudança de cultura subjacente à implementação do LSCM representa alterações nas normas e comportamentos coletivos que englobam a confiança, hierarquia, ambiente de trabalho, comunicação e sentimento de companheirismo (DORA et al., 2016). Um obstáculo recorrente que dificulta a aplicação com sucesso das práticas de LSCM é a resistência das pessoas que são solicitadas a adotar suas práticas e princípios (PEREZ et al., 2010). Neste contexto, justifica-se a alta frequência (76\%) de citação da $\mathrm{B}_{1}$ no portfólio final. Em contrapartida, a barreira menos citada foi $\mathrm{B}_{12}$ (Baixa compreensão dos conceitos e princípios relacionados à implementação do LSCM), com apenas $9 \%$ das citações. Curiosamente, a baixa frequência de citação de tal barreira é contrária ao esperado, já que é essencial a compreensão dos conceitos e princípios do LSCM para realizar a sua implementação (MANZOURI, 2012; MANZOURI et al., 2013a). Anand e Kodali (2008) afirmam que os conceitos relacionados ao LSCM ainda não estão totalmente desenvolvidos, em especial, quanto aos seguintes aspectos: base teórica para o LSCM, elementos que o compõe e o modo de implementação do LSCM. Contudo, justamente devido à falta de estudos que aprofundem tais aspectos do LSCM, o nível de entendimento e percepção quanto à implementação do LSCM ainda é muito raso (MANZOURI et al., 2013a), justificando o desconhecimento e consequentemente baixa citação dessa barreira. 
Tabela 3 - Barreiras de LSCM e sua frequência de citação na literatura (Parte I)

\begin{tabular}{|c|c|c|c|c|c|c|c|c|c|c|c|c|c|c|c|c|c|c|c|c|c|}
\hline & Barreiras/Autores & 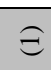 & $\widehat{d}$ & $\widehat{్}$ & 丹 & $\approx$ & e & $E$ & 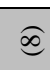 & $\hat{\sigma}$ & $\stackrel{\theta}{\theta}$ & $\Xi$ & $\stackrel{\overbrace{}}{\beth}$ & $\stackrel{(\widetilde{2}}{=}$ & $\stackrel{f}{\Xi}$ & 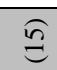 & $\stackrel{6}{\theta}$ & $\stackrel{I}{\Xi}$ & $\stackrel{\infty}{=}$ & $\stackrel{\vartheta}{\triangleq}$ & $\tilde{\Xi}$ \\
\hline $\mathrm{B}_{1}$ & Dificuldades para mudanças culturais & $\mathrm{x}$ & $\mathrm{x}$ & $\mathrm{x}$ & & $\mathrm{x}$ & $\mathrm{x}$ & & $\mathrm{x}$ & & $\mathrm{x}$ & $\mathrm{x}$ & & $\mathrm{x}$ & $\mathrm{x}$ & & $\mathrm{x}$ & $\mathrm{x}$ & $\mathrm{x}$ & & $\mathrm{x}$ \\
\hline $\mathrm{B}_{2}$ & Falta de comprometimento da alta gestão & $\mathrm{x}$ & & & & $\mathrm{x}$ & $\mathrm{x}$ & & $\mathrm{x}$ & $\mathrm{x}$ & $\mathrm{x}$ & $\mathrm{x}$ & $\mathrm{x}$ & $\mathrm{x}$ & & & $\mathrm{x}$ & $\mathrm{x}$ & $\mathrm{x}$ & $\mathrm{x}$ & \\
\hline $\mathrm{B}_{3}$ & Falta de desenvolvimento de equipe especializada & & & & & & & & $\mathrm{x}$ & & $\mathrm{x}$ & & & $\mathrm{x}$ & & & $\mathrm{x}$ & $\mathrm{x}$ & $\mathrm{x}$ & $\mathrm{x}$ & \\
\hline $\mathrm{B}_{4}$ & Falta de confiança para parcerias na cadeia de suprimentos & $\mathrm{x}$ & & $\mathrm{x}$ & $\mathrm{x}$ & $\mathrm{x}$ & & $\mathrm{x}$ & $\mathrm{x}$ & & & & $\mathrm{x}$ & & & & $\mathrm{x}$ & & $\mathrm{x}$ & & \\
\hline $\mathrm{B}_{5}$ & Alta oscilação da demanda & & & & & $\mathrm{x}$ & $\mathrm{x}$ & $\mathrm{x}$ & $\mathrm{x}$ & & $\mathrm{x}$ & & & & $\mathrm{x}$ & $\mathrm{x}$ & $\mathrm{x}$ & & & & \\
\hline $\mathrm{B}_{6}$ & Baixo compartilhamento de informação & & & $\mathrm{x}$ & & $\mathrm{x}$ & & $\mathrm{x}$ & $\mathrm{x}$ & & & & & & & & & $\mathrm{x}$ & & & \\
\hline $\mathrm{B}_{7}$ & Falta de colaboração e envolvimento de toda a cadeia de suprimentos & & & & $\mathrm{x}$ & & $\mathrm{x}$ & $\mathrm{x}$ & $\mathrm{x}$ & $\mathrm{x}$ & & & $\mathrm{x}$ & $\mathrm{x}$ & & & & & & & $\mathrm{x}$ \\
\hline $\mathrm{B}_{8}$ & Falta de disponibilidade de recursos & & & & $\mathrm{x}$ & & & & $\mathrm{x}$ & & & & & & & & & & & $\mathrm{x}$ & \\
\hline $\mathrm{B}_{9}$ & $\begin{array}{l}\text { Ausência de infraestrutura de TIC (Tecnologia de Informação e Comunicação) para } \\
\text { integração }\end{array}$ & & & & & & $\mathrm{x}$ & $\mathrm{x}$ & & & & & $\mathrm{x}$ & $\mathrm{x}$ & & & & & $\mathrm{x}$ & & \\
\hline $\mathrm{B}_{10}$ & Resistência para aderir estratégias de longo prazo & & & $\mathrm{x}$ & & & & & & & & & & & $\mathrm{x}$ & $\mathrm{x}$ & $\mathrm{x}$ & $\mathrm{x}$ & $\mathrm{x}$ & & \\
\hline $\mathrm{B}_{11}$ & Complexidade da cadeia de suprimentos & $\mathrm{x}$ & & & & & & $\mathrm{x}$ & & & & $\mathrm{x}$ & & & $\mathrm{x}$ & $\mathrm{x}$ & & & & & \\
\hline & $\begin{array}{l}\text { Baixa compreensão dos conceitos e princípios relacionados à implementação do } \\
\text { LSCM }\end{array}$ & & & & & & & & & & & & & $\mathrm{x}$ & & & & & & $\mathrm{x}$ & \\
\hline
\end{tabular}

Autores: (1) Lamming, 1996; (2) Erridge e Murray, 1998; (3) McIvor, 2001; (4) Arkader, 2001; (5) Huang et al., 2002; (6) Yew Wong et al., 2005; (7) Jaklic et al., 2006; (8) Taylor, 2006; (9) Eisler et al., 2007; (10) Morgan, 2007; (11) Machado e Pereira, 2008; (12) Adamides et al., 2008; (13) Anand e Kodali, 2008; (14) Wee e Wu, 2009; (15) Stavrulaki e Davis, 2010; (16) Perez et al., 2010; (17) Cudney e Elrod, 2011; (18) Behrouzi e Wong, 2011; (19) Manzouri, 2012; (20) Azevedo et al., 2012.

Tabela 3 - Barreiras de LSCM e sua frequência de citação na literatura (Parte II)

\begin{tabular}{|c|c|c|c|c|c|c|c|c|c|c|c|c|c|c|c|c|}
\hline & Barreiras/Autores & $\widehat{\bar{d}}$ & తิ & త્తి & $\stackrel{f}{d}$ & $\sqrt{d}$ & d्d & $\widehat{\overparen{d}}$ & 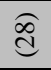 & ¿ิ & क्रे & ले & ฮิ & ల్రి & है & $\begin{array}{l}\text { Frequência de } \\
\text { citação }\end{array}$ \\
\hline $\mathrm{B}_{1}$ & Dificuldades para mudanças culturais & $\mathrm{x}$ & $\mathrm{x}$ & $\mathrm{x}$ & $\mathrm{x}$ & $\mathrm{x}$ & $\mathrm{x}$ & & $\mathrm{x}$ & & $\mathrm{x}$ & $\mathrm{x}$ & $\mathrm{x}$ & $\mathrm{x}$ & $\mathrm{x}$ & 26 \\
\hline $\mathrm{B}_{2}$ & Falta de comprometimento da alta gestão & & $\mathrm{x}$ & $\mathrm{x}$ & $\mathrm{x}$ & & $\mathrm{x}$ & $\mathrm{x}$ & & $\mathrm{x}$ & $\mathrm{x}$ & & $\mathrm{x}$ & $\mathrm{x}$ & & 22 \\
\hline $\mathrm{B}_{3}$ & Falta de desenvolvimento de equipe especializada & $\mathrm{x}$ & $\mathrm{x}$ & $\mathrm{x}$ & $\mathrm{x}$ & $\mathrm{x}$ & $\mathrm{x}$ & $\mathrm{x}$ & $\mathrm{x}$ & $\mathrm{x}$ & $\mathrm{x}$ & $\mathrm{x}$ & $\mathrm{x}$ & $\mathrm{x}$ & $\mathrm{x}$ & 21 \\
\hline $\mathrm{B}_{4}$ & Falta de confiança para parcerias na cadeia de suprimentos & & $\mathrm{x}$ & & $\mathrm{x}$ & & $\mathrm{x}$ & $\mathrm{x}$ & & & & $\mathrm{x}$ & & & & 14 \\
\hline $\mathrm{B}_{5}$ & Alta oscilação da demanda & $\mathrm{x}$ & & & & & $\mathrm{x}$ & & & & & & & & $\mathrm{x}$ & 11 \\
\hline $\mathrm{B}_{6}$ & Baixo compartilhamento de informação & & & $\mathrm{x}$ & $\mathrm{x}$ & & & $\mathrm{x}$ & & & $\mathrm{x}$ & & $\mathrm{x}$ & & $\mathrm{x}$ & 11 \\
\hline $\mathrm{B}_{7}$ & Falta de colaboração e envolvimento de toda a cadeia de suprimentos & & & $\mathrm{x}$ & $\mathrm{x}$ & & & & & & & & & & & 10 \\
\hline $\mathrm{B}_{8}$ & Falta de disponibilidade de recursos & $\mathrm{x}$ & $\mathrm{x}$ & & $\mathrm{x}$ & & & $\mathrm{x}$ & & & $\mathrm{x}$ & & $\mathrm{x}$ & & $\mathrm{x}$ & 10 \\
\hline $\mathrm{B}_{9}$ & $\begin{array}{l}\text { Ausência de infraestrutura de TIC (Tecnologia de Informação e Comunicação) para } \\
\text { integração }\end{array}$ & & & $\mathrm{x}$ & & & & $\mathrm{x}$ & & & $\mathrm{x}$ & & $\mathrm{x}$ & $\mathrm{x}$ & & 10 \\
\hline $\mathrm{B}_{10}$ & Resistência para aderir estratégias de longo prazo & & & & & & & & & & & & & & & 6 \\
\hline $\mathrm{B}_{11}$ & Complexidade da cadeia de suprimentos & & $\mathrm{x}$ & & & & & & & & & & & & & 6 \\
\hline $\mathrm{B}_{12}$ & Baixa compreensão dos conceitos e princípios relacionados à implementação do LSCM & & $\mathrm{x}$ & & & & & & & & & & & & & 3 \\
\hline
\end{tabular}

2014; (27) Manzouri et al., 2014b; (28) Tortorella et al., 2015; (29) Vlachos, 2015; (30) Adebanjo et al., 2016; (31) Jajja et al., 2016; (32) Dora et al., 2016; (33) Kumar et al., 2016; (34) Bevilacqua et al., 2017. 


\subsection{Variáveis contextuais para a implementação do LSCM}

Fatores contextuais são aspectos ou elementos que significam que o desempenho de um sistema de gestão é condicionado pelas características específicas de uma empresa ou seu ambiente, tais como número de funcionários, volumes de vendas, setor, tempo em que um sistema de gestão é implementado e assim por diante. As variáveis contextuais representam características situacionais geralmente exógenas à organização focal ou gestora (TORTORELLA et al., 2015). Uma série de fatores contextuais são inerentes à cada cadeia de suprimentos e afetam a relação entre a cooperação de seus membros e seu desempenho (GUEIMONDE-CANTO et al., 2011). Contudo, a modificação dessas variáveis tende a ser limitada e somente possível com um esforço a longo prazo (MANZOURI et al., 2013a). Assim, levar em consideração a influência desses fatores é de vital importância para uma melhor compreensão da implementação do LSCM (CAMACHO-MIÑANO et al., 2013).

$\mathrm{Na}$ Tabela 4 foram compilados os fatores contextuais (FC) inerentes à implementação do LSCM de acordo com o portfólio final de artigos. A partir dos 60 artigos revisados, apenas 30 abordaram explicitamente algum FC. Observa-se que, além de existir um grau variável de frequência referente a cada um dos FC, a frequência total de citações e a quantidade de artigos que abordaram sobre o assunto foi significativamente inferior àqueles que trataram sobre as práticas e barreiras. $\mathrm{O}$ fator contextual $\mathrm{FC}_{1}$ (Tamanho da empresa), mostrou-se como o fator mais citado quanto sua influência sobre a implementação do LSCM, com 63\% de citações. Hadid e Afshin Mansouri (2014) comentam que organizações de grande porte podem ter maiores níveis de adoção de práticas de LSCM, uma vez que normalmente possuem redes de cadeias de suprimentos mais complexas e necessitam maior eficiência na sua gestão. Em contrapartida, o fator contextual $\mathrm{FC}_{8}$ (Volume de produção) foi o menos citado dentro da literatura analisada, com apenas $7 \%$ de citações. Esse fato pode ser decorrente da incipiência de estudos que abordem os FC no contexto das cadeias de suprimentos. 
Tabela 4 - Fatores Contextuais de LSCM e sua frequência de citação na literatura

\begin{tabular}{|c|c|c|c|c|c|c|c|c|c|c|c|c|c|c|c|c|c|c|c|c|c|c|c|c|c|c|c|c|c|c|c|c|}
\hline & Autores & $€$ & d & $\widehat{(ల)}$ & త & $\approx$ & e & $E$ & $\widehat{\infty}$ & $\widehat{\varrho}$ & $\stackrel{\varrho}{\ominus}$ & $\Xi$ & $\overparen{\Xi}$ & $\stackrel{\overparen{\Omega}}{=}$ & $\underset{\Xi}{\mathcal{\Xi}}$ & $\stackrel{n}{\approx}$ & $\stackrel{6}{=}$ & $\triangleq$ & $\stackrel{\infty}{=}$ & $\stackrel{\partial}{\triangleq}$ & త్రి & $\widehat{\bar{d}}$ & త్ర & $\widehat{\overparen{d}}$ & $\underset{d}{\stackrel{f}{d}}$ & $\stackrel{\mathfrak{d}}{\mathfrak{d}}$ & $\stackrel{d}{d}$ & $\widehat{\overparen{d}}$ & 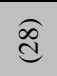 & $\widehat{\grave{d}}$ & હิ & $\begin{array}{l}\text { Frequência } \\
\text { de citação }\end{array}$ \\
\hline $\mathrm{FC}_{1}$ & Tamanho da empresa & & & $\mathrm{x}$ & & & $\mathrm{x}$ & & $\mathrm{x}$ & $\mathrm{x}$ & $\mathrm{x}$ & $\mathrm{x}$ & & $\mathrm{x}$ & $\mathrm{x}$ & & $\mathrm{x}$ & $\mathrm{x}$ & $\mathrm{x}$ & & & $\mathrm{x}$ & $\mathrm{x}$ & $\mathrm{x}$ & $\mathrm{x}$ & & $\mathrm{x}$ & $\mathrm{x}$ & $\mathrm{x}$ & & $\mathrm{x}$ & 19 \\
\hline $\mathrm{FC}_{2}$ & Equipe multifuncional treinada & & $\mathrm{x}$ & & $\mathrm{x}$ & & $\mathrm{x}$ & & & & $\mathrm{x}$ & & $\mathrm{x}$ & $\mathrm{x}$ & & & $\mathrm{x}$ & & & & & & $\mathrm{x}$ & & $\mathrm{x}$ & & & $\mathrm{x}$ & & $\mathrm{x}$ & & 11 \\
\hline $\mathrm{FC}_{3}$ & Localização geográfica & $\mathrm{x}$ & & $\mathrm{x}$ & & $\mathrm{x}$ & & $\mathrm{x}$ & & & & & $\mathrm{x}$ & $\mathrm{x}$ & & $\mathrm{x}$ & & & & & $\mathrm{x}$ & & & & & $\mathrm{x}$ & $\mathrm{x}$ & & & & & 10 \\
\hline $\mathrm{FC}_{4}$ & Segmento da cadeia & & & & & & & & $\mathrm{x}$ & & & & $\mathrm{x}$ & $\mathrm{x}$ & & $\mathrm{x}$ & & $\mathrm{x}$ & $\mathrm{x}$ & & & & & & & $\mathrm{x}$ & & $\mathrm{x}$ & & & & 8 \\
\hline $\mathrm{FC}_{5}$ & Fatores econômicos do país & $\mathrm{x}$ & & $\mathrm{x}$ & & & & $\mathrm{x}$ & & & & & & & & $\mathrm{x}$ & & $\mathrm{x}$ & $\mathrm{x}$ & & & & & & & & $\mathrm{x}$ & & $\mathrm{x}$ & & & 8 \\
\hline $\mathrm{FC}_{6}$ & Nível educacional & $\mathrm{x}$ & $\mathrm{x}$ & & & & & & & & $\mathrm{x}$ & & & & & & $\mathrm{x}$ & & & $\mathrm{x}$ & & & & & $\mathrm{x}$ & & & & & & & 6 \\
\hline $\mathrm{FC}_{7}$ & Idade da planta & & & & & & & & & & & & & & & & & & & & & $\mathrm{x}$ & & & $\mathrm{x}$ & & $\mathrm{x}$ & & & & & 3 \\
\hline $\mathrm{FC}_{8}$ & Volume de produção & & & & & & & & & & & & & & & & $\mathrm{x}$ & & & & & & & & & & & $\mathrm{x}$ & & & & 2 \\
\hline
\end{tabular}

Autores: (1) Arkader, 2001; (2) Huang et al., 2002; (3) Taylor, 2006; (4) Machado e Pereira, 2008; (5) Adamides et al., 2008; (6) Anand e Kodali, 2008; (7) Found et al., 2008; (8) Qi e Chu, 2009; (9) Wee e Wu, 2009; (10) Perez et al., 2010; (11) Rahman et al.; 2010; (12) Gueimonde-Canto et al., 2011; (13) Cudney e Elrod, 2011; (14) Manzouri, 2012; (15) Azevedo et al., 2012; (16) Karim e Arif-Uz-Zaman, 2013; (17) CamachoMiñano et al., 2013; (18) Manzouri et al., 2013a; (19) Martínez-Jurado e Moyano-Fuentes, 2014; (20) Jadhav et al., 2014; (21) Hadid e Afshin Mansouri, 2014; (22) Bhamu e Singh Sangwan, 2014; (23) Manzouri et al., 2014b; (24) Tortorella et al., 2015; (25) Adebanjo et al., 2016; (26) Jajja et al. 2016 ; (27) Dora et al., 2016; (28) Marodin et al., 2016; (29) Duarte e Machado, 2017; (30) Bevilacqua et al., 2017. 


\section{Conclusão}

Grande parte dos trabalhos publicados até hoje sobre LSCM concentrou-se em delinear práticas enxutas e seus potenciais benefícios, inferindo que uma vez que as empresas as adotem, a implementação enxuta estaria automaticamente iniciada. Porém, na realidade, tal implementação em toda a extensão da cadeia de suprimentos é extremamente difícil e desafiadora.

O presente estudo procurou identificar as principais práticas, barreiras e fatores contextuais inerentes à implementação do LSCM. Para atingir o objetivo, foi feito um levantamento sistemático da literatura identificando-se um portfólio com 60 artigos, de modo a apresentar um panorama do tema. Cabe destacar que a maioria das pesquisas abordam os três tópicos (práticas, barreiras e fatores contextuais) de modo isolado, não correlacionando-os. Assim, este estudo contribui para o fortalecimento do corpo de conhecimento sobre LSCM, identificando 18 práticas, 12 barreiras e 8 fatores contextuais inerentes a sua implementação, e os enumerando de forma a proporcionar uma análise conjunta.

Desse modo, algumas sugestões para trabalhos futuros são propostas a partir de lacunas de pesquisa identificadas, sendo elas: o desenvolvimento de estudos que identifiquem, classifiquem e validem empiricamente as principais práticas de LSCM, de modo a direcionar a construção de um conceito consolidado. $\mathrm{O}$ estudo também apresentou as principais barreiras inerentes à implementação das práticas de LSCM e concluiu que adotar medidas preventivas podem ajudar a mitigar as barreiras para implementação do LSCM típicas do contexto no qual a cadeia de suprimentos está inserida. Assim, investigar o efeito moderador das variáveis contextuais da cadeia de suprimentos sobre o relacionamento entre as barreiras e as práticas de LSCM caracteriza uma oportunidade para estudos futuros na área. Por fim, destaca-se a incipiência dos estudos relacionados aos métodos de avaliação de maturidade das cadeias de suprimentos quanto ao nível de implementação LSCM. Logo, a definição de metodologias que forneçam subsídio para avaliar de uma forma abrangente a cadeia produtiva envolvendo todos os seus agentes apresenta-se como uma oportunidade de pesquisa.

\section{Referências}

ADAMIDES, E. D. et al. Supporting collaboration in the development and management of lean supply networks. Production Planning and Control, v. 19, n. 1, p. 35-52, 2008.

ADEBANJO, D. et al. Prioritizing lean supply chain management initiatives in healthcare service operations: a fuzzy AHP approach. Production Planning \& Control, v. 27, n. 12, p. 953-966, 2016.

AFONSO, H.; CABRITA, M. do R. Developing a lean supply chain performance framework in a SME: a perspective based on the balanced scorecard. Procedia Engineering, v. 131, p. 270-279, 2015.

AL-AOMAR, R. A lean construction framework with six sigma rating. International Journal of Lean Six Sigma, v. 3, n. 4, p. 299-314, 2012.

ALVES FILHO, A. G. et al. Pressupostos da gestão da cadeia de suprimentos: evidências de estudos sobre a indústria automobilística. Revista Gestão e Produção, v. 11, n.3, p. 275-288, 2004.

ANAND, G.; R. KODALI. A conceptual framework for lean supply chain and its implementation. International Journal of Value Chain Management, v. 2, n. 3, p. 313-357, 2008.

ARIF-UZ-ZAMAN, K.; AHSAN, A. M. M. N. Lean supply chain performance measurement. International Journal of Productivity and Performance Management, v. 63, n. 5, p. 588-612, 2014. 
ARKADER, R. The perspective of suppliers on lean supply in a developing country context. Integrated Manufacturing Systems, v. 12, n. 2, p. 87-93, 2001.

AZEVEDO, S. G. et al. Influence of green and lean upstream supply chain management practices on business sustainability. IEEE Transactions on Engineering Management, v. 59, n. 4, p. 753-765, 2012.

BALLOU, R. H. Gerenciamento da cadeia de suprimentos/logística empresarial. 5 ed. Porto Alegre: Bookman, 2006, 616 p.

BAKER, P. Aligning distribution center operations to supply chain strategy. The International Journal of Logistics Management, v. 15, n. 1, p. 111-123, 2004.

BEVILACQUA, M. et al. Relationships between Italian companies' operational characteristics and business growth in high and low lean performers. Journal of Manufacturing Technology Management, v. 28, n. 2, p. 250-274, 2017.

BEHROUZI, F.; WONG, K. Y. An investigation and identification of lean supply chain performance measures in the automotive SMEs. Scientific Research and Essays, v. 6, n. 24, p. 5239-5252, 2011.

BHAMU, J.; SINGH SANGWAN, K. Lean manufacturing: literature review and research issues. International Journal of Operations \& Production Management, v. 34, n. 7, p. 876-940, 2014.

BIRGÜN BARLA, S. A case study of supplier selection for lean supply by using a mathematical model. Logistics Information Management, v. 16, n. 6, p. 451-459, 2003.

BOONSTHONSATIT, K.; JUNGTHAWAN, S. Lean supply chain management-based value stream mapping in a case of Thailand automotive industry. IEEE International Conference on Advanced logistics and Transport, p. 65-69, 2015.

CAMACHO-MIÑANO, M. et al. What can we learn from the evolution of research on lean management assessment?. International Journal of Production Research, v. 51, n. 4, p. 1098-1116, 2013.

CARVALHO, H. et al. Lean, agile, resilient and green: divergencies and synergies. International Journal of Lean Six Sigma, v. 2, n. 2, p. 151-179, 2011 a.

CARVALHO, $\mathrm{H}$. et al. Modelling green and lean supply chains: an eco-efficiency perspective. Resources, Conservation and Recycling, v. 120, p. 75-87, $2017 \mathrm{~b}$.

CHRISTOPHER, M.; TOWILL, D. An integrated model for the design of agile supply chains. International Journal of Physical Distribution and Logistics Management, v. 31, n. 4, p. 235-246, 2001.

CUDNEY, E.; ELROD, C. A comparative analysis of integrating lean concepts into supply chain management in manufacturing and service industries. International Journal of Lean Six Sigma, v. 2, n. 1, p. 5-22, 2011.

DORA, M. et al. Determinants and barriers to lean implementation in food-processing SMEs-a multiple case analysis. Production Planning \& Control, v. 27, n. 1, p. 1-23, 2016.

DROHOMERETSKI, E. et al. Lean supply chain management: practices and performance measures. In: IIE Annual Conference. Proceedings. Institute of Industrial and Systems Engineers, p. 1, 2012. 
DUARTE, S. et al. Green and lean implementation: an assessment in the automotive industry. International Journal of Lean Six Sigma, v. 8, n. 1, p. 65-88, 2017.

DÜES, C. M. et al. Green as the new lean: how to use lean practices as a catalyst to greening your supply chain. Journal of cleaner production, v. 40, p. 93-100, 2013.

EISLER, M, et al. Cooperation of lean enterprises - Techniques used for lean supply chain. In: Advances in Production Management Systems, Springer US, p. 363-370, 2007.

ERRIDGE, A.; MURRAY, J. G. The application of lean supply in local government: the Belfast experiments. European Journal of Purchasing \& Supply Management, v. 4, n. 4, p. 207-221, 1998.

FOUND, P. et al. Creating a sustainable lean business system within a multi-national group company. In: IIE Annual Conference. Proceedings. Institute of Industrial Engineers-Publisher, p. 302, 2008.

GUEIMONDE-CANTO, A. et al. Competitive effects of co-operation with suppliers and buyers in the sawmill industry. Journal of Business \& Industrial Marketing, v. 26, n. 1, p. 58-69, 2011.

HADID, W; AFSHIN MANSOURI, S. The lean-performance relationship in services: a theoretical model. International Journal of Operations \& Production Management, v. 34, n. 6, p. 750-785, 2014.

HARTONO, Y. et al. Enabler to Successful Implementation of Lean Supply Chain in a Book Publisher. Procedia Manufacturing, v. 4, p. 192-199, 2015.

HINES, P. et al. Learning to evolve: a review of contemporary lean thinking. International journal of operations \& production management, v. 24, n. 10, p. 994-1011, 2004.

HUANG, S. H. et al. A product driven approach to manufacturing supply chain selection. Supply Chain Management: An International Journal, v. 7, n. 4, p. 189-199, 2002.

JADHAV, J. R. et al. Exploring barriers in lean implementation. International Journal of Lean Six Sigma, v. 5, n. 2, p. 122-148, 2014.

JAJJA, M. S. S et al. Supply chain strategy and the role of suppliers: evidence from the Indian subcontinent. Benchmarking: An International Journal, v. 23, n. 7, p. 1658-1676, 2016.

JAKLIC, J. et al. Enhancing lean supply chain maturity with business process management. Journal of information and organizational sciences, v. 30, n. 2, p. 205-223, 2006.

JASTI, N. V. K.; KODALI, R. Lean production: literature review and trends. International Journal of Production Research, v. 53, n. 3, p. 867-885, 2015 a.

JASTI, N. V. K.; KODALI, R. A critical review of lean supply chain management frameworks: proposed framework. Production Planning \& Control: The Management of Operations, v. 26, p. 1051-1068, 2015b.

KARIM, A; ARIF-UZ-ZAMAN, K. A methodology for effective implementation of lean strategies and its performance evaluation in manufacturing organizations. Business Process Management Journal, v. 19, n. 1, p. 169-196, 2013.

KUMAR, S. et al. Barriers in green lean six sigma product development process: an ISM approach. Production Planning \& Control, v. 27, n. 7-8, p. 604-620, 2016. 
LA LONDE, B. J. et al. Integrated distribution systems: a management perspective. International Journal of Physical Distribution, v. 1, n. 1, p. 43-49, 1971.

LAMMING, R. Squaring lean supply with supply chain management. International Journal of Operations \& Production Management. v. 16, n. 2, p. 183-196, 1996.

LANCASTER, F. W. Indexação e resumos: teoria e prática. Tradução de Antônio Agenor Briquet de Lemos. rev. atual. 2004.

LEVY, D. L. Lean production in an international supply chain. Sloan management review, v. 38, n. 2, p. 94, 1997.

LI, S.; RAGU-NATHAN, B.; RAGU-NATHAN, T. S.; RAO, S. S. The impact of supply chain management practices on competitive advantage and organizational performance. The International Journal of Management Science (Omega), v. 34, n. 2, p. 107-124, 2006.

MACHADO, V. C.; PEREIRA, A. Modelling lean performance. In: Management of innovation and technology, 2008. ICMIT 2008. 4th IEEE international conference on. IEEE, p. 1308-1312, 2008.

MANZOURI, M. How lean supply chain implementation affect halal food companies. Advances in Natural and Applied Sciences, v. 6, n. 8, p. 1485-1490, 2012.

MANZOURI, M.; RAHMAN, M. N. Ab. Adaptation of theories of supply chain management to the lean supply chain management. International Journal of Logistics Systems and Management, v. 14, n. 1, p. 38-54, 2013.

MANZOURI, M. et al. Lean supply chain practices in the Halal food. International Journal of Lean Six Sigma, v. 4, n. 4, p. 389-408, 2013 a.

MANZOURI, M. et al. Increasing production and eliminating waste through lean tools and techniques for halal food companies. Sustainability, v. 6, n. 12, p. 9179-9204, 2014b.

MARODIN, G. A. et al. Contextual factors and lean production implementation in the Brazilian automotive supply chain. Supply Chain Management: An International Journal, v. 21, n. 4, p. 417-432, 2016.

MARTÍNEZ-JURADO, P. J.; MOYANO-FUENTES, J. Lean management, supply chain management and sustainability: a literature review. Journal of Cleaner Production, v. 85, p. 134-150, 2014.

MCIVOR, R. Lean supply: the design and cost reduction dimensions. European Journal of Purchasing \& Supply Management, v. 7, n. 4, p. 227-242, 2001.

MORGAN, C. Supply network performance measurement: future challenges?. The International Journal of Logistics Management, v. 18, n. 2, p. 255-273, 2007.

OLESEN, P. et al. Using lean principles to drive operational improvements in intermodal container facilities: A conceptual framework. Journal of Facilities Management, v. 13, n. 3, p. 266-281, 2015.

PARÉ, G.; TRUDEL, M. C.; JAANA, M.; KITSIOU, S. Synthesizing information systems knowledge: A typology of literature reviews. Information \& Management, v. 52, n. 2, p. 183-199, 2015.

PARVEEN, C. M.; RAO, TVVLN. An integrated approach to design and analysis of lean manufacturing system: a perspective of lean supply chain. International Journal of Services and Operations Management, v. 5, n. 2, p. 175-208, 2009. 
PARVEEN, C. M. et al. Integration of lean and green supply chain-Impact on manufacturing firms in improving environmental efficiencies. In: Green Technology and Environmental Conservation, 2011. GTEC 2011. International Conference on. IEEE, p. 143-147, 2011.

QI, Yi-nan; CHU, Zhao-fang. The impact of supply chain strategies on supply chain integration. In: Management Science and Engineering, 2009. ICMSE 2009. International Conference on. IEEE, p. 534-540, 2009.

QRUNFLEH, S.; TARAFDAR, M. Lean and agile supply chain strategies and supply chain responsiveness: the role of strategic supplier partnership and postponement. Supply Chain Management: An International Journal, v. 18, n. 6, p. 571-582, 2013.

RAHMAN, S. et al. Impact of lean strategy on operational performance: a study of Thai manufacturing companies. Journal of manufacturing technology management, v. 21, n. 7, p. 839$852,2010$.

SHAH, R.; WARD, P. T. Defining and developing measures of lean production. Journal of operations management, v. 25, n. 4, p. 785-805, 2007.

SHARMA, V. et al. Impact of lean practices on performance measures in context to Indian machine tool industry. Journal of Manufacturing Technology Management, v. 26, n. 8, p. 1218-1242, 2015.

SONI, G.; KODALI, R. Interpretive structural modeling and path analysis for proposed framework of lean supply chain in Indian manufacturing industry. Journal of Industrial and Production Engineering, v. 33, n. 8, p. 501-515, 2016.

TORTORELLA, G. L. et al. The impact of contextual variables on learning organization in firms that are implementing lean: a study in Southern Brazil. The International Journal of Advanced Manufacturing Technology, v. 78, n. 9-12, p. 1879-1892, 2015.

VILAÇA, M. L. C. Pesquisa e ensino: considerações e reflexões. Revista e-scrita: Revista do Curso de Letras da UNIABEU, v. 1, n. 2, p. 59-74, 2010.

VLACHOS, I. Applying lean thinking in the food supply chains: a case study. Production Planning \& Control, v. 26, n. 16, p. 1351-1367, 2015.

WIENGARTEN, F. et al. Exploring synergetic effects between investments in environmental and quality/lean practices in supply chains. Supply Chain Management: An International Journal, v. 18, n. 2, p. 148-160, 2013. 